\title{
EPIDEMIOLOGICAL RELATIONSHIP BETWEEN HORMONE REPLACEMENT THERAPY AND BREAST CANCER
}

Amanda Cristina de Souza Vieira', Antônio Pedro Oliveira de Vasconcelos¹, Jaqueline Maria Pinheiro de Araujo', Juliana Comin Müller ${ }^{1}$, Larissa de Cassia Afonso Magalhães ${ }^{1}$, Renato Duarte da Silva' ${ }^{1}$ Ricardo Baroni Vieira ${ }^{1}$, Fabiana Candida de Queiroz Santos Anjos ${ }^{1}$

'Universidade de Gurupi - Gurupi (TO), Brazil.

Objective: The aim of this study was to epidemiologically analyze the hormone replacement therapy (HRT) and the emergence of breast cancer. Methodology: This is an article review from the databases such as LILACS, SciELO, Bireme, and Medscape, utilizing the keywords: menopause, HRT, breast cancer and complications, employing the use of connectors when necessary. This review aims to elucidate what has been published in the last years about the usage of hormone therapy and the emergence of breast cancer since there are disagreements between the literature. Results: The analysis displayed positive effects during the usage of HRT, such as maintenance of bone density, prevention of fractures, and cardiovascular events in patients with no previous changes in this system, and also showed us a strong relationship between HRT and the incidence of breast cancer in menopausal women with a focus into the imposing time of use ratio. Meanwhile, this development risk of breast CA can be reduced in the long run with the withdrawal of the previously initiated therapy. The progesterone HRT has been shown to have lower risks in association with estrogen than when compared with the association of synthetic progestins and estrogen. Associations have similar results for oral and skin HRT. In patients using the postmenopausal hormone therapy, the risk of mortality from breast cancer was reduced in patients with exposure for a maximum of 5 years, more than 5-10 years, or more than 10 years. Conclusion: In view of the exposure, it is considered that the HRT is more beneficial than malefic to the life and health of women. Meanwhile, the risk of breast CA goes up while the HRT time stretches over the years. Thus, it is necessary to individually evaluate the benefits and risks to better identify the therapy that should be utilized.

Keywords: Menopause; Hormone Replacement Therapy; Breast Cancer and Complications. 